\title{
A Methodical Block based Feature Level Image Fusion Technique with Wavelet Transform using Neural Network for Satellite Images
}

\author{
T. Prabhakara Rao, B. Rama Rao
}

\begin{abstract}
This paper describe about the feature extraction or detection machine learning application which one is wavelet transform integrated with neural network. It has obtained an effective block based feature level with wavelet transform using neural network (BFWN) model for image fusion. In the projected $B F W N$ model, the discrete wavelet transform (DWT) and neural network (NN) are considered for fusing IRS-1D images using LISS- III scanner about the location different areas in India. Also Quick Bird image data and Landsat 7 image data are used to carry out on the proposed BFWN method. The characteristics like contrast visibility, energy of gradient, spatial frequency, variance and edge information are under study. A Feed forward back propagation neural network is trained and tested for categorization since the learning capability of neural network makes it feasible to customize the image fusion process. The trained neural network is used to fuse the two source images. The proposed BFWN model is distinguish, with DWT alone to assess the quality of the fused image. The results obviously show that the proposed BFWN model is a capable and feasible algorithm for image fusion.
\end{abstract}

Keywords--- Image Fusion, DWT, Neural Network, Block Based Features, Performance Measures, Discrete Wavelet Transform, Neural Network.

\section{INTRODUCTION}

In recent years, image processing has gained so much significance because of its extended applications in different fields like satellite imagery, health care, military etc. However, despite of its deep development possibilities, enormous size data (image) amount comes along which cause severe hurdle in terms of storage and efficiency of processing.

Big Data is a moving target, what is considered to be "Big" today will not be so years ahead. The Big Data was initially defined with 3Vs: Volume, Velocity, and Variety. At present people are talking about $7 \mathrm{Vs}$ where the other $4 \mathrm{Vs}$ are: Veracity, Value, Visualization, and Variability [1]. Only $90 \%$ of the world's data has been created which consists of $80 \%$ unstructured data moreover videos and photos [2]. Millions of user's requests for image searching, storage and querying in different domains. Since In the last two years, around two million CCTV cameras are in use which records around 25 billion hours of video per year in countries like UK. Fig. 1 shows how the images/videos are related to Big Data, which we are calling as Big Video / Image Data.

Revised Version Manuscript Received on August 14, 2019.

T. Prabhakara Rao, Research Scholar, Shri Venkateswara University, Gajraula, Uttar Pradesh, India. E-mail: prabhakar.tatpudi@gmail.com

Dr.B. Rama Rao, Professor, Shri Venkateswara University, Gajraula, Uttar Pradesh, India. E-mail: drbrrao2015@gmail.com
Big Image Data (BID) Processing is all about processing two categories of images. The first one is about dealing with large number of images i.e. Quantitative [3]. The second one is about processing gigantic images. Here gigantic means either based on the dimension of a single image where a single image may of size in Gig pixels [4] or based on the size where a single image may occupy Giga Bytes of memory in computer. Mainly the image processing tools and programs run on a single machine. This single machine also has batch processing system, but limited capability of memory is some disadvantage with this single-processing machine. So, a new approach is essential to process the data parallel. This drawback motivates the need of new application that do process parallel and give efficiency in processing of larger image data sets [5]

The present Technique extends the concepts of previous Technique by proposing an integrated approach of image fusion with wavelet transform using neural networks. To address this and to improve quality of the fused image, the present idea integrates Discrete Wavelet Transform (DWT) with Neural Network (NN), which is one of machine learning applications the feature extraction or detection. The present method derived 'An effective Block Based Feature Level Image Fusion Technique with Wavelet Transform using Neural Network' (BFWN). In the proposed BFWN model, 'Discrete Wavelet Transform' (DWT) is integrated with 'Neural Network' (NN) for fusing Indian Remote Sensing satellite (IRS-1D), Landsat-7, and Quick Bird images. The fusion of Multispectral (MS) and Panchromatic (PAN) images is performed to retrieve complementary information from the source images. The proposed BFWN model used the idea of NN to train the neurons based upon the retrieved features. Feed forward back propagation neural network is trained and tested for classification since the learning capability of NN makes it feasible to customize the image fusion process. To fuse the pair of source images the trained $\mathrm{NN}$ is then used. The proposed BFWN models differentiate with DWT.

\section{IMAGE FUSION WITH WAVELET TRANSFORMATION}

Image fusion with Wavelet transformations provides multi scale and multi resolution analysis functions. Wavelet transform is a linear tool in its original form [6].

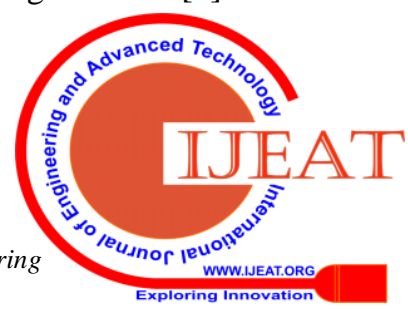




\section{A METHODICAL BLOCK BASED FEATURE LEVEL IMAGE FUSION TECHNIQUE WITH WAVELET TRANSFORM USING NEURAL NETWORK FOR SATELLITE IMAGES}

Wavelet technique performs better than the standard fusion technique in spectral and spatial quality. The Decimated wavelet transformations are Discrete Wavelet Transformations (DWT)[7] and the Undecimated Wavelet Transformations are Stationary Wavelet Transformations (SWT). Wavelets are three general classes they are Orthogonal, Bi orthogonal and Non orthogonal in both SWT and DWT. The Wavelet filters used for Orthogonal wavelets are Haar and Daubachies, for Biorthogonal wavelets are Bior1.1, Bior1.3, Bior1.7 and for No orthogonal wavelets are Meyers, Coiflets and Symlets. The 2-D discrete wavelet transformation is used for fusing images. The five wavelet fusion methods are discussed In this paper i.e., orthogonal wavelet fusion with decimation (ORTH), top-hat transform at, biorthogonal wavelet fusion with decimation (BIOR), orthogonal wavelet fusion with undecimation (UORTH), and biorthogonal wavelet fusion with undecimation (UBIOR). A. Additive based Image Fusion method Perform histogram match process between MS and PAN images to obtain three new PAN images. Use the Wavelet Transform to decompose new PAN images and different bands of MS image. Add the detail images of the decomposed PAN image at different levels to the corresponding details of different bands in the MS image and acquire the new details component in the different bands of the MS image. Perform
Inverse Wavelet Transform on the bands of MS images respectively and get the fused image [8].

DWT is superior to the earlier standard methods and also pyramid-based methods since DWT does not carry redundant information across different resolutions and provides directional information. And also many different selection fusion rules were proposed to fuse wavelet coefficients. The learning capability of neural networks makes it feasible to customize the image fusion process, as it is a good classification method and acts as a self-organizing expert system. The classification accuracy of remote sensing images is improved when multiple source image data are introduced to the processing [9].Hence, DWT and neural networks are integrated to exploit the classification capabilities of neural networks to fuse high resolution panchromatic image and low resolution Multispectral image. The combination of several different fusion schemes approved to be a useful strategy to achieve better quality of results $[10,11]$.

\section{PROPOSED BFWN METHOD FOR IMAGE FUSION}

The block diagram of the proposed BFWN method is shown in Figure 1.

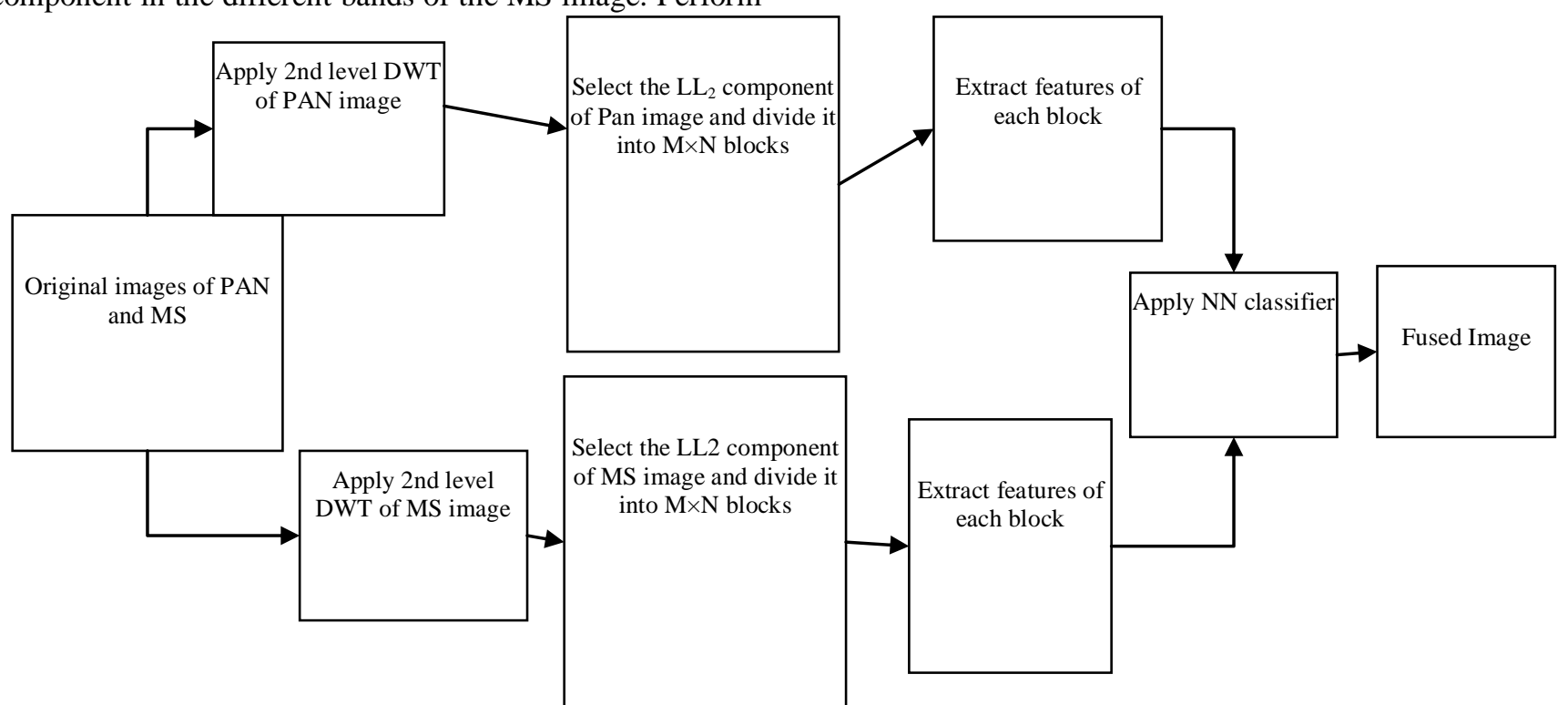

Figure 1: Block Diagram of the Proposed BFWN Method

The stepwise working of the proposed BFWN algorithm is described below.

1. Read MS and PAN as Source images.

2. Apply MWT at 2nd level decomposition to the two images.

3. Consider the $\mathrm{LL}_{2}$ component of PAN and MS images.

4. Partition $\mathrm{LL}_{2}$ component of each image into nonoverlapped blocks of size $4 \times 4$ or $8 \times 8$.

5. Extract statistical features (such as contrast visibility, energy of gradient, spatial frequency, variance and edge information) from each block of PAN and MS images. These features are treated as feature vector $F_{1}$ of PAN image and feature vector $\mathrm{F}_{2}$ of MS image.

6. Subtract the feature values of $F_{1}$ and $F_{2}$ of each block. The difference between $F_{1}$ and $F_{2}$ if 0 then

denote it as 1 else -1 . Then construct an index vector (i.e. the combination of 1 's and -1 's).

7. Index vector is given to the classifier for classification which will be given as an input to the NN.

8. Train the newly constructed NN randomly by simulating it.

9. Consider corresponding block of PAN image if the simulated output $>1$ else consider corresponding block of MS image.

10. By selecting the appropriate block from step 9 Construct the fused image. 
11. Some quality measures are required to assess the quality of fused image. In the present work, the quality assessment is derived on BFWN method using image quality metrics such as mean squared error (MSE), standard deviation (SD), entropy, correlation coefficient (CC), peak signal to noise ratio (PSNR), root mean squared error (RMSE), mean absolute error (MAE), mutual information measure (MIM), fusion factor(FF) and the metric $\mathrm{QAB} / \mathrm{F}$ are used. These quality metrics are discussed in the following Equations from (1) to (16).

Standard Deviation (SD): It is used to measure the level of contrast in the fused image. Standard Deviation is calculated by the Equation (1).

$$
S D=\sqrt{\sum_{i=0}^{L}\left(i-i^{\prime}\right)^{2} h_{F}(i)}
$$

Where $i^{\prime}=\sum_{i=0}^{L} i h_{F}$ and $h_{F}$ is the normalized histogram of Fused image $L$ is the number of gray levels. A well contrast image will have high standard deviation.

Entropy: It is used in the quantitative analysis and evaluation image details; the entropy value is used as it provides better comparison of the image [12]. It also shows the probability distribution of pixels with the distinct grey values. A higher value shows good fusion results. It is calculated by the Equation (2).

$$
H=-\sum_{i=0}^{L-1} h_{F}(i) \log _{2} h_{F}(i)
$$

Where $\mathrm{hF}$ is the normalized histogram of the fused image and $\mathrm{L}$ is the number of gray levels.

Correlation Coefficient (CC): Pearson's CC is denoted by ' $r$ ', and is used for comparing the source image and the fused image. If $r=1$ then the two images are absolutely identical, if $r=0$ then they are completely uncorrelated and if $\mathrm{r}=-1$ then they are completely anti-correlated. It is calculated by using Equation (3).

$$
r=\frac{\sum_{i}\left(x_{i}-x_{m}\right)\left(y_{i}-y_{m}\right)}{\sqrt{\sum_{i}\left(x_{i}-x_{m}\right)^{2}} \sqrt{\sum_{i}\left(y_{i}-y_{m}\right)^{2}}}
$$

Where $x i$ is the intensity of the $i_{\text {th }}$ pixel in the source image, $y_{i}$ is the intensity of the $i_{\text {th }}$ pixel in the fused image, $x_{m}$ is the mean intensity of the source image and $y_{m}$ is the mean intensity of the fused image.

Mean Squared Error (MSE): the spectral distortion in the fused image, is measured by MSE. A smaller value shows good fusion results. It is calculated by using Equation (4).

$$
M S E=\frac{\sum_{i=1}^{M} \sum_{j=1}^{N}\left(I_{R}(i, j)-I_{F}(i, j)^{2}\right)}{M * N}
$$

Where $I_{R}(i, j)$ indicates pixel $(i, j)$ of the image reference $I_{F}(i, j)$ Indicates pixel $(i, j)$ of the fuse image,

$M * N$ is size of the image.

Peak Signal to Noise Ratio (PSNR): PSNR is used to reveal the radiometric distortion of the fused image compared with original image. A higher value shows good fusion results. It is defined by using the Equation (5).

$$
\operatorname{PSNR}(d B)=10 \log _{10}\left(\frac{255 * 255}{\mathrm{MSE}}\right)
$$

Root Mean Squared Error (RMSE): RMSE is used to measure the standard error in the fused image [13]. A smaller value shows good fusion results. It is calculated by using Equation (6).

$$
R M S E=\sqrt{M S E}
$$

Mean Absolute Error (MAE): It is used to measure the average magnitude of errors in a set of forecasts, without considering their direction. It measures precision for continuous variables. A smaller value shows good fusion results. It is calculated by using Equation (7).

$$
M A E=\frac{1}{M * N} \sum_{x=0}^{M-1} \sum_{y=0}^{N-1}\left(I_{R}(x, y)-I_{F}(x, y)\right)
$$

Where $I_{R}(x, y)$ is a pixel $(x, y)$ of the image reference, $I_{F}(x, y)$ is a pixel $(x, y)$ of the fuse image with the image size of $\mathrm{M} * \mathrm{~N}$.

Mutual Information Measure (MIM): MIM measures the similarity of image intensity distribution of the corresponding image pair. The greatest value shows the better fusion result. The mutual information between the source image $\mathrm{A}$ and the fused image $\mathrm{F}$ is denoted by IAF and defined by using Equation 8).

$$
I_{A F}=\sum_{a, f} P_{A F}(a, f) \log \frac{P_{A F}(a, f)}{P_{A}(a) P_{F}(f)}
$$

Where PAF is the jointly normalized histogram of $\mathrm{A}$ and $\mathrm{F}, \mathrm{PA}$ and PF are the normalized histogram of $\mathrm{A}$ and $\mathrm{F}$, and $\mathrm{a}$ and $\mathrm{f}$ represent the pixel value of the image $\mathrm{A}$ and $\mathrm{F}$ respectively.

The mutual information between the source image $\mathrm{B}$ and the fused image $\mathrm{F}$ is denoted by IBF and is calculated similarly as IAF. The combined information between the source images A, B and the fused image $\mathrm{F}$ is given by Equation (9). Larger MIM value indicates better fusion result.

$$
M_{F}^{A B}=I_{A F}+I_{B F}
$$

Fusion Factor (FF): A higher value of Fusion Factor indicates that fused image contains moderately good amount of information present in both the images. It is defined by using the Equation (10).

$$
\mathrm{FF}=\mathrm{I}_{\mathrm{AF}}+\mathrm{I}_{\mathrm{BF}}
$$

Where $\mathrm{A}$ and $\mathrm{B}$ are the source images and $\mathrm{F}$ is the fused image.

The metric QAB/F: QAB/F evaluates the amount of edge information transferred from source images into fused image. The greatest value shows the better fusion result. It is defined by using Equation (11).

$$
Q^{A B / F}=\frac{\sum_{n=1}^{N} \sum_{m=1}^{M}\left(Q^{A F}(n, m) w^{A}(n, m)+Q^{B F}(n, m) w^{B}(n, m)\right)}{\sum_{n=1}^{N} \sum_{m=1}^{M}\left(w^{A}(n, m)+w^{B}(n, m)\right)}
$$

Where $Q^{A F}(n, m)=Q_{g}^{A F}(n, m) Q_{\propto}^{A F}(n, m) ; Q_{g}^{A F}(n, m)$ and $Q_{\propto}^{A F}(n, m)$ are the edge strength and orientation preservation values respectively; $\mathrm{n}$ and $\mathrm{m}$ represents the image location; and $\mathrm{N}, \mathrm{M}$ are the size of images respectively. $Q^{B F}(n, m)$ is similar to $Q^{A F}(n, m) \cdot w^{A}(n, m)$ and $w^{B}(n, m)$ reflect the importance of $Q^{A F}(n, m)$ and $Q^{B F}(n, m)$ respectively.

The dynamic range of $\mathrm{QAB} / \mathrm{F}$ is $[0,1]$ and it should be as close to 1 as possible.

\section{RESULTS AND DISCUSSIONS}

The experiments are conducted on IRS-1D images using LISS-III scanner about the locations Hyderabad, Vishakhapatnam, Mahaboobnagar and Patancheru in Andhra Pradesh, India. The Landsat-7 and Quick Bird image datasets are also experimented.

The following Figures (2) to (7) show the fused images of six locations namely Hyderabad, Vishakhapatnam, Mahaboobnagar, Patancheru, Landsat-7 and Quick Bird of the proposed BFWN method. 

TRANSFORM USING NEURAL NETWORK FOR SATELLITE IMAGES

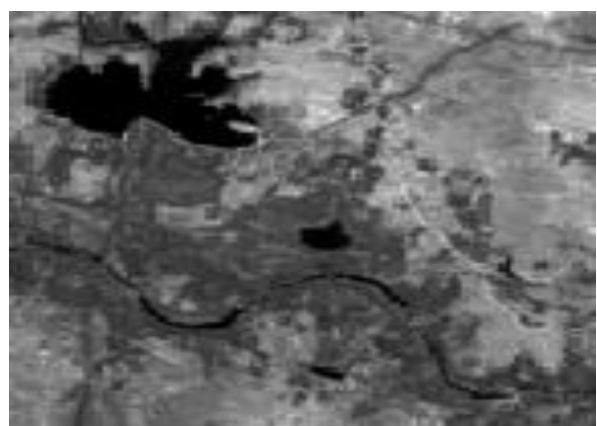

(a)

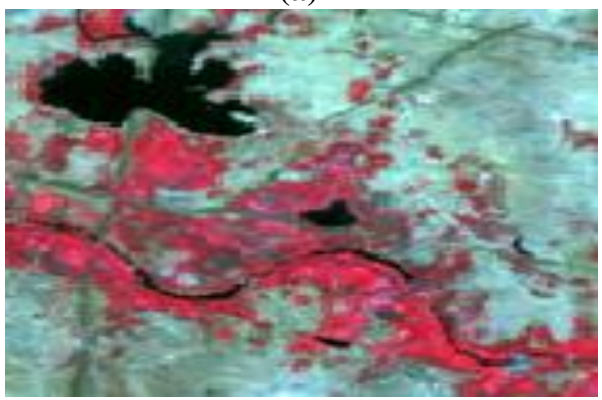

(b)

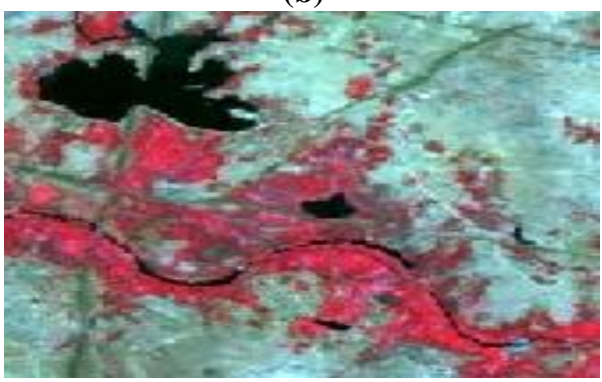

(c)

Figure 2: Location of Hyderabad, (a) PAN Image (b) MS Image and (c) Fused Image Using the Proposed BFWN Method

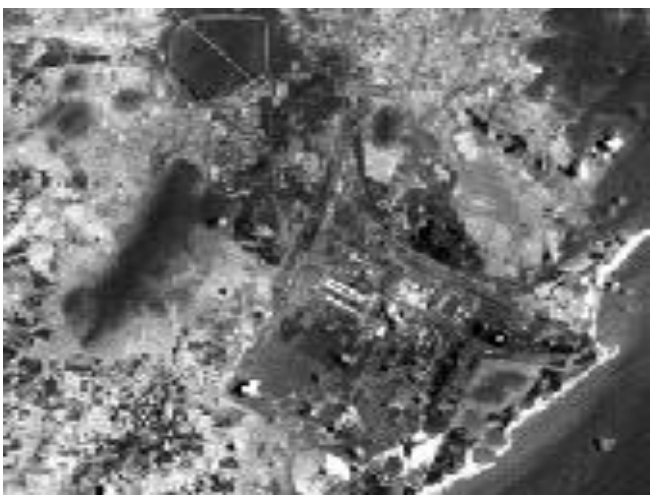

(a)

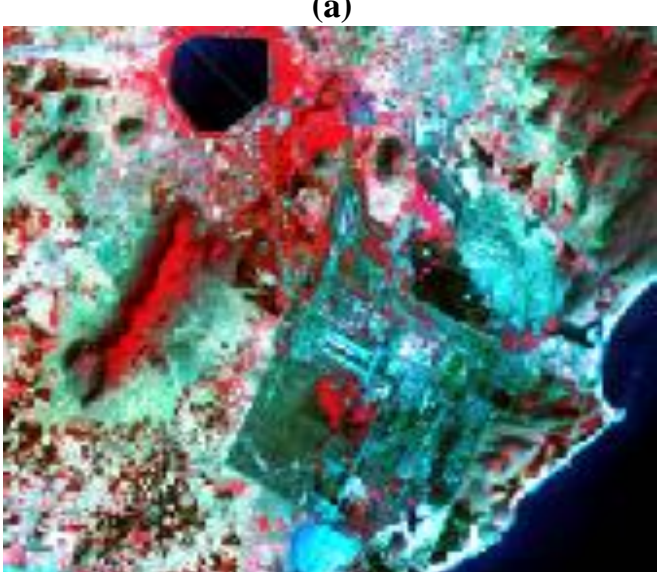

(b)

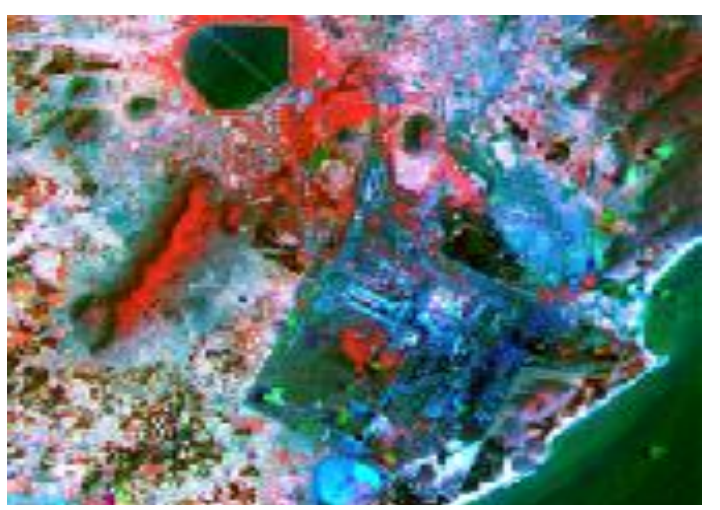

(c)

Figure 3: Location of Visakhapatnam (a) PAN Image (b) MS Image and (c) Fused Image Using the Proposed BFWN Method

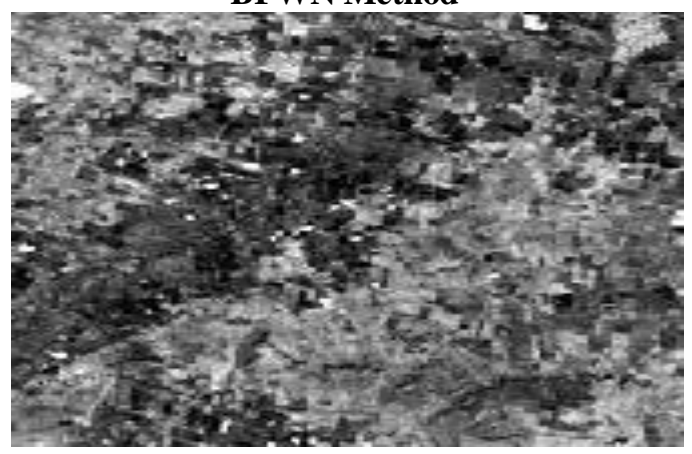

(a)

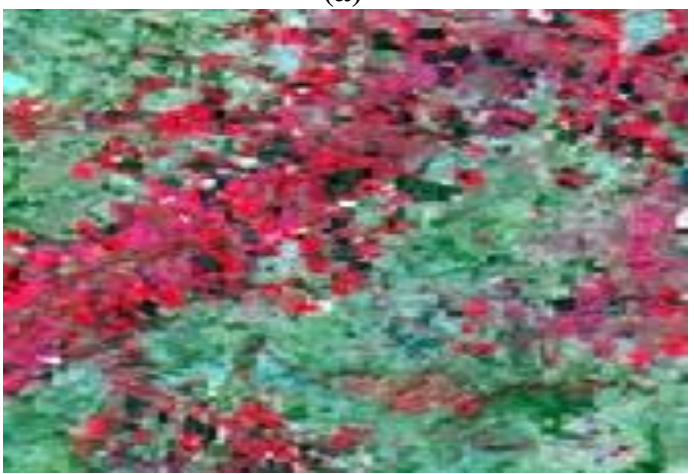

(b)

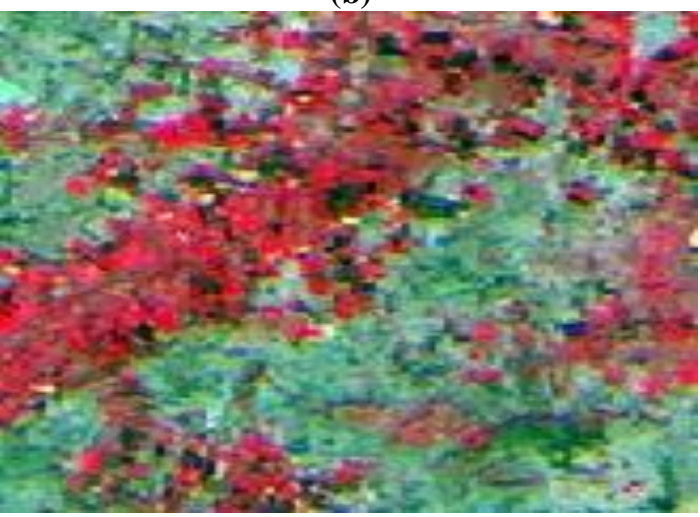

(c)

Figure 4: Location of Mahaboobnagar (a) PAN Image (b) MS Image and (c) Fused Image Using the Proposed BFWN Method

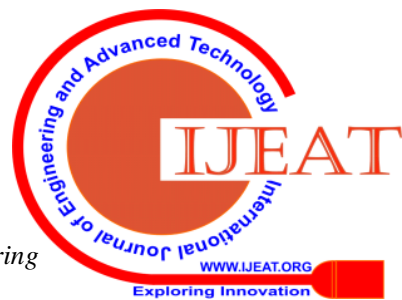




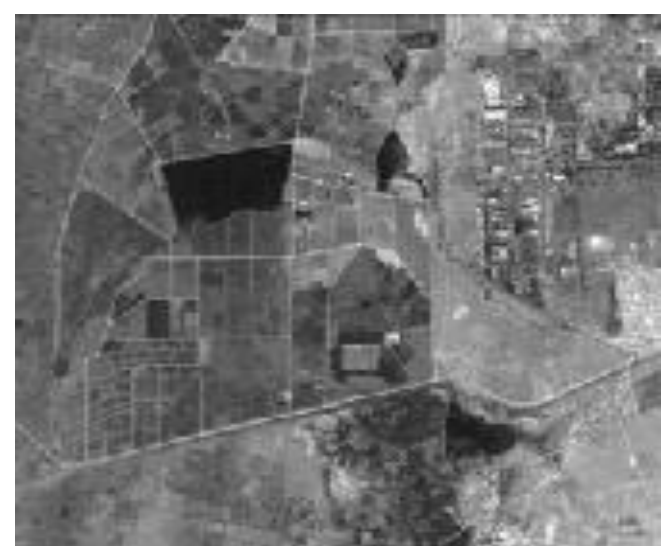

(a)

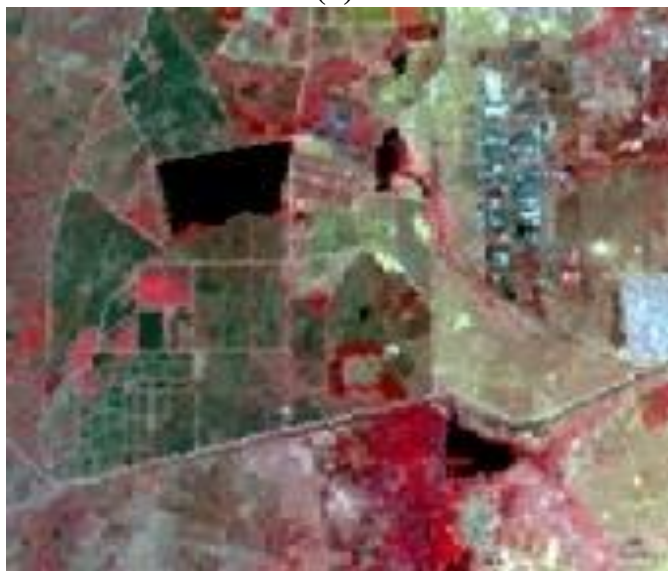

(b)

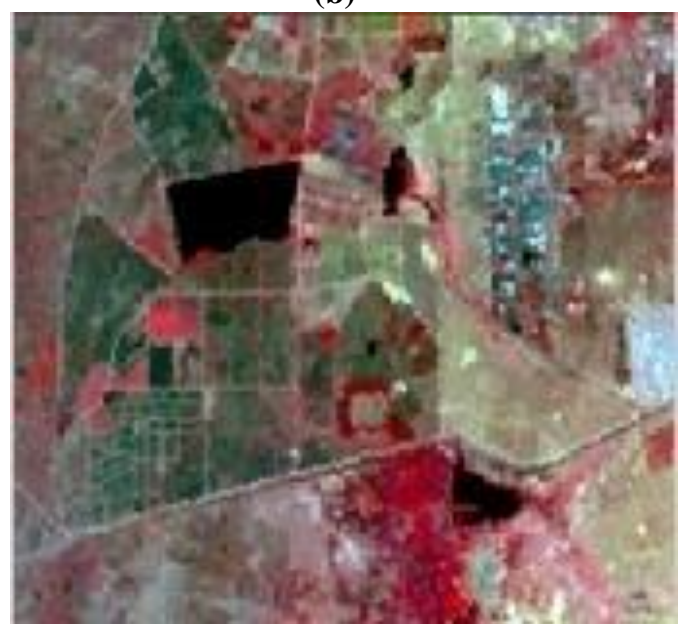

(c)

Figure 5: Location of Patancheru (a) PAN Image (b) MS Image and (c) Fused Image Using the Proposed BFWN Method

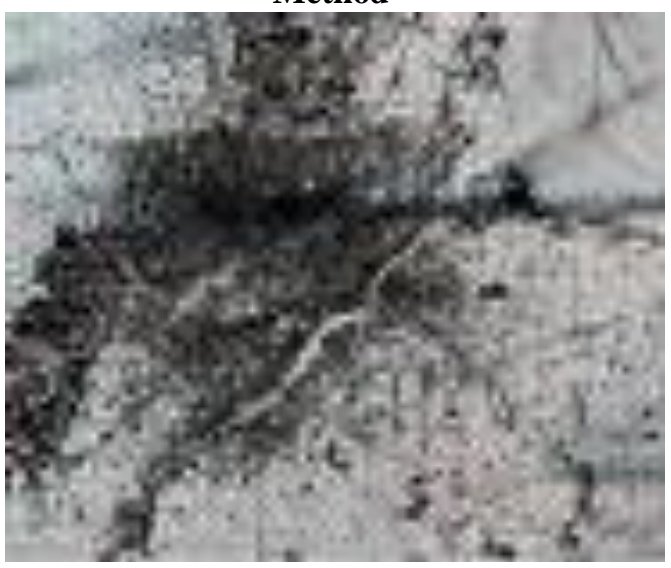

(a)

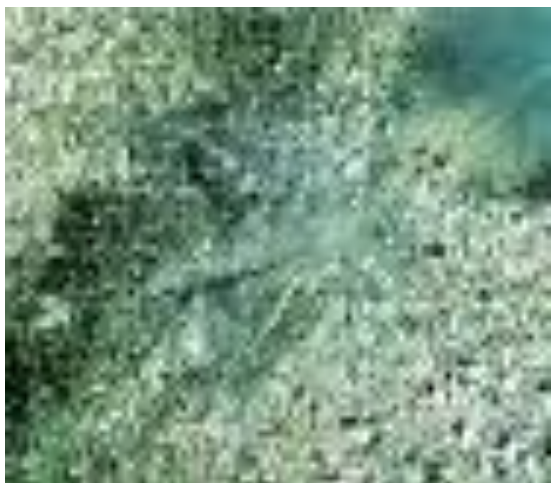

(b)

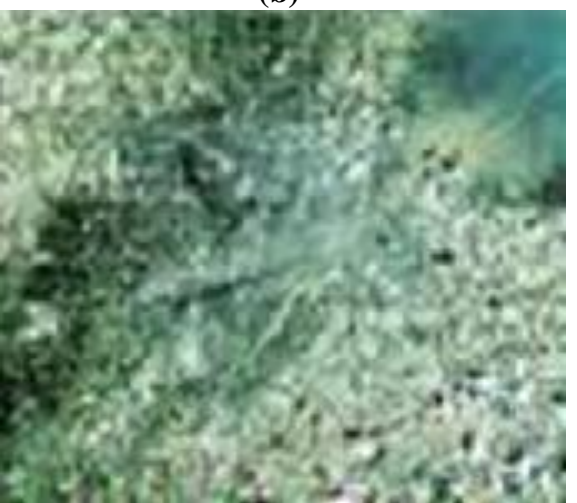

(c)

Figure 6: Location of Landsat-7 (a) PAN Image (b) MS Image and (c) Fused Image using the Proposed BFWN Method

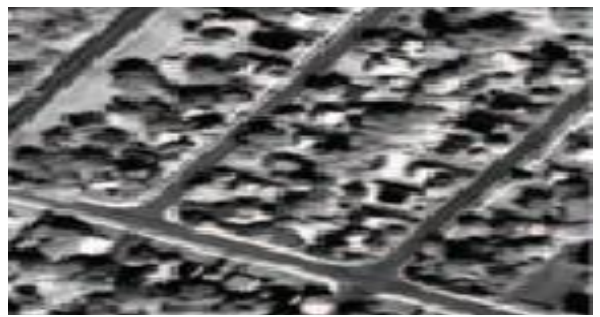

(a)

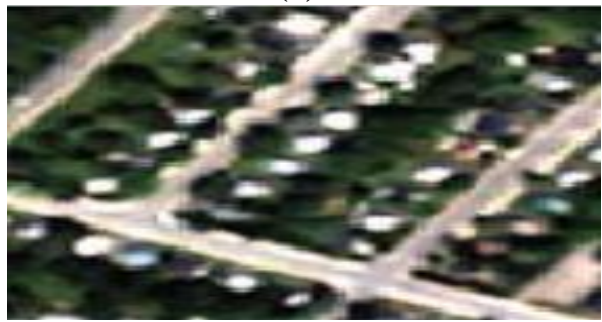

(b)

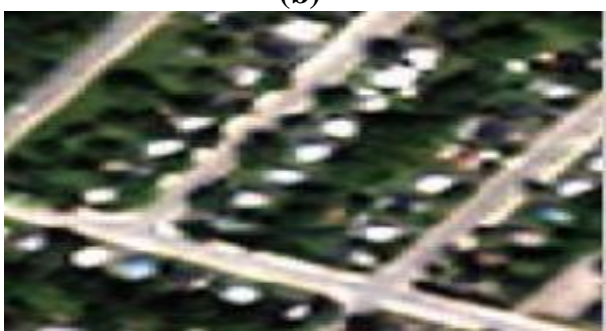

(c)

Figure 7: Location of Quick Bird (a) PAN image (b) Ms Image and (c) Fused Image Using the Proposed BFWN Method

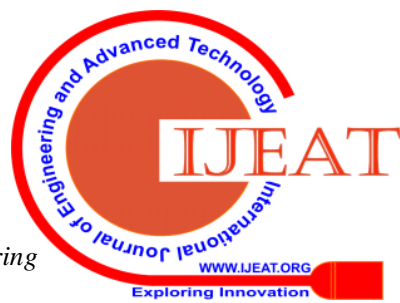




\section{A METHODICAL BLOCK BASED FEATURE LEVEL IMAGE FUSION TECHNIQUE WITH WAVELET TRANSFORM USING NEURAL NETWORK FOR SATELLITE IMAGES}

The Table 1 shows the results of quality metrics using the proposed BFWN method for all the above six locations. By observing the results of Table 1 of the proposed BFWN method, the following observations are made. The average value of correlation coefficient $(\mathrm{CC})$ is almost $\cong 1$, which implies that the fused image is similar to the corresponding original MS image.

The higher correlation between the high frequency components of the fusion PAN image indicates that more spatial information from the PAN image is injected into the fusion result. The average value of Peak Signal to Noise Ratio (PSNR) is greater than 70, which implies that the spectral information of MS image and high signal is preserved most effectively and it is more robust. The average value of Entropy is above 7, which implies that the fused image contains rich information and better quality than either of the source images. The average value of Mutual Information Measure (MIM) is greater than 1, which indicates that good amount of information of the source images is furnished in the fused image. The average value of Fusion Factor (FF) is above 2, which indicates that the similarity of image intensity distribution of the corresponding image pair is induced in the fused image. The average value of $\mathrm{QAB} / \mathrm{F}$ is around 0.5 , which indicates that good amount of edge information is transferred from the source images to the fused image. The average value of Standard Deviation (SD) is around 50, which implies that not much deviation is induced in the fused image. The average value of Mean Squared Error (MSE) is less than 0.1 , which indicates that the spectral distortion in the fused image is comparatively less.

The average value of Root Mean Squared Error (RMSE) is less than 0.1, which indicates that less standard error is induced in the fused image. If the average value of Mean Absolute Error (MAE) is less than 0.1, then it indicates that less average magnitude of the errors in a set of forecasts is induced in the fused image.

Table 1: Quality Metrics Using Proposed BFWN Method about the Locations Hyderabad, Vishakhapatnam, Mahaboobnagar, Patancheru, Landsat-7 and Quick Bird

\begin{tabular}{|l|l|l|l|l|l|l|l|}
\hline Metrics & Hyderabad & Vishakhapatnam & Mahaboobnagar & Patancheru & Landsat-7 & Quick Bird & Average \\
\hline MSE & 0.0013 & 0.0058 & 0.0038 & 0.0027 & 0.0029 & 0.0019 & 0.0031 \\
\hline Ent & 7.1384 & 7.8438 & 7.688 & 7.3715 & 7.4416 & 7.7809 & 7.544 \\
\hline CC & 0.998 & 0.9992 & 0.9995 & 0.9994 & 0.998 & 0.998 & 0.9987 \\
\hline SD & 40.5197 & 62.3872 & 49.444 & 35.4804 & 39.6275 & 77.5713 & 50.8384 \\
\hline PSNR & 77.0658 & 70.5083 & 72.3011 & 73.8269 & 74789 & 75.4508 & 7772 \\
\hline RMSE & 0.0357 & 0.0761 & 0.0619 & 0.0519 & 0.054 & 0.0431 & 0.0538 \\
\hline MAE & 0.0161 & 0.0357 & 0.0291 & 0.0258 & 0.0296 & 0.0194 & 0.026 \\
\hline MIM & 2.0289 & 1.0235 & 1.006 & 1.1985 & 1.6842 & 1.8487 & 1.465 \\
\hline FF & 4.0577 & 2.047 & 2.012 & 2.397 & 3684 & 6974 & 2.9299 \\
\hline QAB/F & 0.3801 & 0.5925 & 0.4107 & 0.5221 & 0.323 & 0.3019 & 0.4217 \\
\hline
\end{tabular}

Figure 8 and Figure 9 below show fused images of all the six locations of PAN and MS images using the existing DWT fusion technique and the proposed BFWN method.

By observing the results of Figure.8 and Figure 9, according to the visual perception, it is clear that the fused images using DWT are having more of blurring effects compared to the fused images using the proposed BFWN method. As $\mathrm{NN}$ is a good classifying technique, it trains the classification more number of times which results in sharpening the fused image. The proposed BFWN method is distinguish, with DWT and methods proposed by Siddiqui et al. [14], Luo et al. [15], Yuhendra [16], Khosravi et al. [17], and Zheng et al [18].

After in-depth literature survey, the present study found that no method was experimented by considering all the quality metrics which are considered in the present study. Among the considered metrics in the present study, the method proposed by Siddiqui et al. [14], considered the parameters SD, entropy, PSNR and RMSE. The method proposed by Luo et al. [15], considered the parameter CC. The method proposed by Yuhendra [16], considered the parameters MSE, RMSE and MAE. The method proposed by Khosravi et al. [17], considered the parameters MIM and $\mathrm{QAB} / \mathrm{F}$.

The method proposed by Zheng et al. [18], considered the parameters Entropy, PSNR and RMSE. Hence, the present study conducted experiments on the above proposed methods and derived the quality metrics considered in the present study on the image data sets Hyderabad, Vishakhapatnam, Mahaboobnagar, Patancheru, Landsat-7 and Quick Bird.

Table 2 depicts the calculated average metric values and average calculated statistical quality metrics of the proposed BFWN technique with the existing DWT fusion technique and other image fusion techniques proposed by some other authors. 
Table 2: Quality Metrics of the Proposed BFWN Method with Other Image Fusion Methods

\begin{tabular}{|l|l|l|l|l|l|l|l|}
\hline Metrics & DWT & Siddiqui et al., & Luo et al., & Yuhendra & Khosravi et al., & Zheng et al., & BFWN \\
\hline SD & 56.8824 & 45.5710 & 51.3320 & 52.3498 & 60.3413 & 52.4356 & 50.8384 \\
\hline ENT & 7.5303 & 7.1234 & 7.5030 & 7.2498 & 7.0932 & 7.1584 & 7.5440 \\
\hline CC & 0.9305 & 0.9023 & 0.9097 & 0.9117 & 0.9580 & 0.9627 & 0.9987 \\
\hline MSE & 0.0049 & 0.0041 & 0.0036 & 0.0081 & 0.0176 & 0.0106 & 0.0031 \\
\hline PSNR & 71.623 & 62.3150 & 47836 & 71.3422 & 40.8756 & 39.4065 & 7772 \\
\hline RMSE & 0.0684 & 0.1967 & 4.2453 & 9.0498 & 1.2874 & 2.8590 & 0.0538 \\
\hline MAE & 0.0312 & 1.0435 & 0.1839 & 6.9300 & 0.0732 & 0.0590 & 0.0260 \\
\hline MIM & 0.774 & 0.980 & 0.5640 & 0.983 & 0.2315 & 0.9803 & 1.4650 \\
\hline FF & 1.548 & 1.3245 & 0.4397 & 0.3452 & 0.0987 & 1.2342 & 2.9299 \\
\hline QAB/F & 0.36 & 0.0234 & 0.134 & 0.4029 & 0.0309 & 0.0118 & 0.4217 \\
\hline
\end{tabular}

The first column of Table 2 represents the names of the quality metrics evaluated for the present study. The second column represents the values of the existing fusion technique, DWT.

The third column represents the values of the method proposed by Siddiqui et al.[14]. The fourth column represents the values of the method proposed by Luo et al. [15]. The fifth column represents the values of the method proposed by Yuhendra [16]. The sixth column represents the values of the method proposed by Khosravi et al. [17]. The sixth column represents the values of the method proposed by Zheng et al. [18]. The eighth column represents the values of the proposed BFWN method. It is observed that the average values of Entropy, CC, PSNR, MIM, FF and $\mathrm{QAB} / \mathrm{F}$ are high for the proposed BFWN method compared to the above considered methods mentioned in Table 2.

Also, it is observed that the average values of SD, MSE, RMSE and MAE are low for the proposed BFWN method compared to the above considered methods mentioned in Table 2.

Figure 10 presents a graph giving the comparative analysis of the proposed BFWN method with the other existing methods about the quality parameters.

It clearly indicates that the average values of the metric parameters Entropy, CC, PSNR, MIM, FF and QAB/F yields higher value for the proposed BFWN method and the average values of the metric parameters SD, MSE, RMSE and MAE yields smaller value for the proposed BFWN method.

The above two observations indicate that the fused image using the proposed BFWN method is similar to the corresponding original MS image and contains more spatial information from the PAN image.

In the fused image, high signal is preserved and it is more robust. It also indicates that good amount of edge information is transferred and the spectral distortions are comparatively reduced. Hence, it is ascertained that the proposed BFWN method out performs other methods which are compared in Table 2.

For fusing the considered six pairs of data images using the existing DWT and the proposed BFWN method, the comparison of PSNR values is depicted in Figure 11, the comparison of SD values is depicted in Figure 12, the comparison of CC values is depicted in Figure 13, and the comparison of MIM values is depicted in Figure 14.
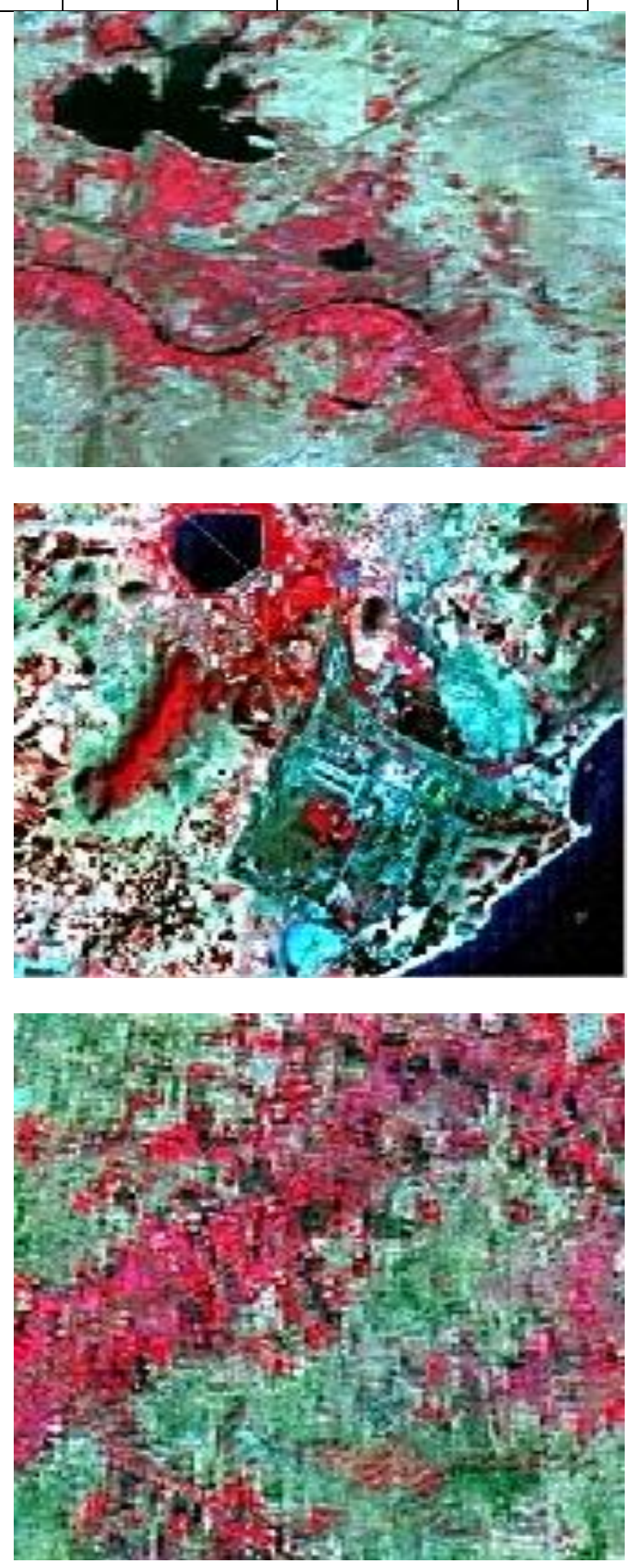

TRANSFORM USING NEURAL NETWORK FOR SATELLITE IMAGES
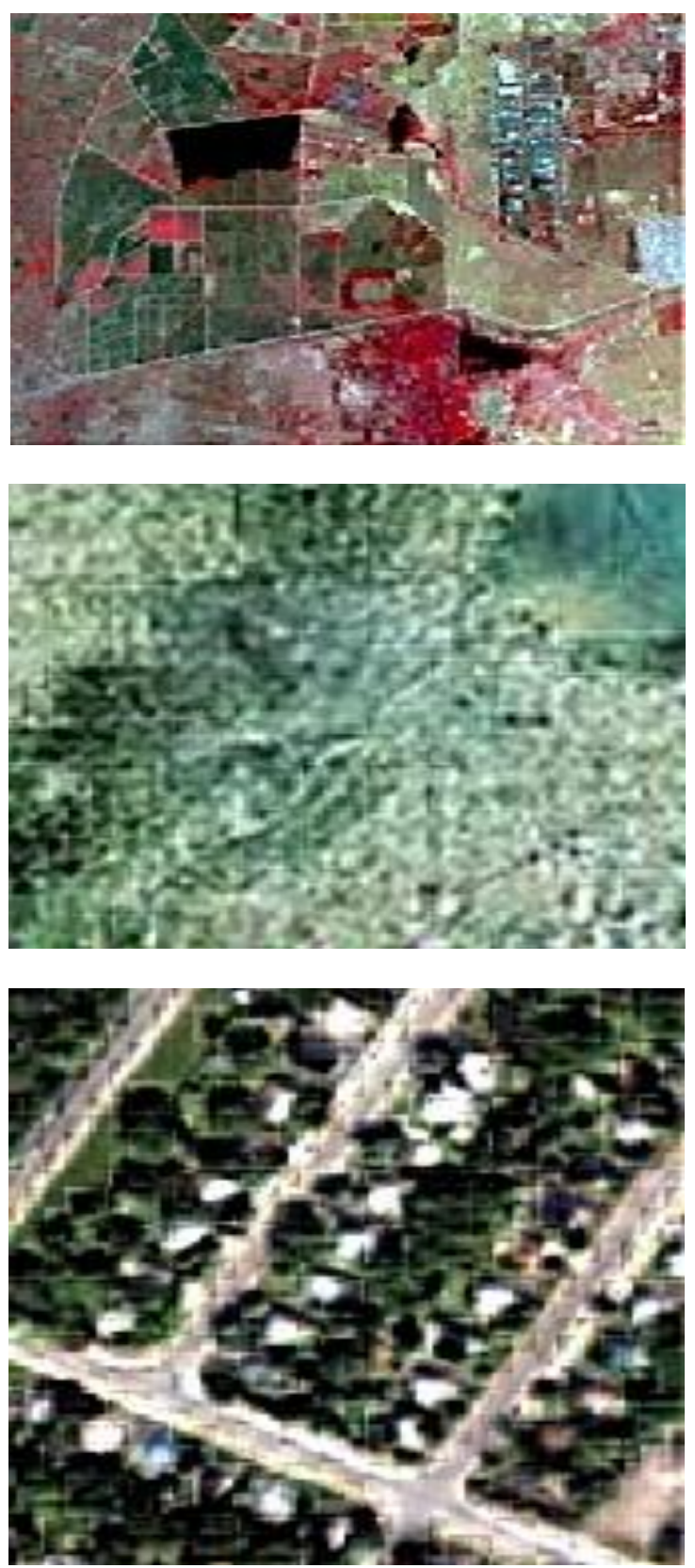

Figure 8: Existing DWT Fusion Technique at the Locations Hyderabad, Visakhapatnam, Mahaboobnagar, Patancheru, Landsat-7 and Quick Bird.

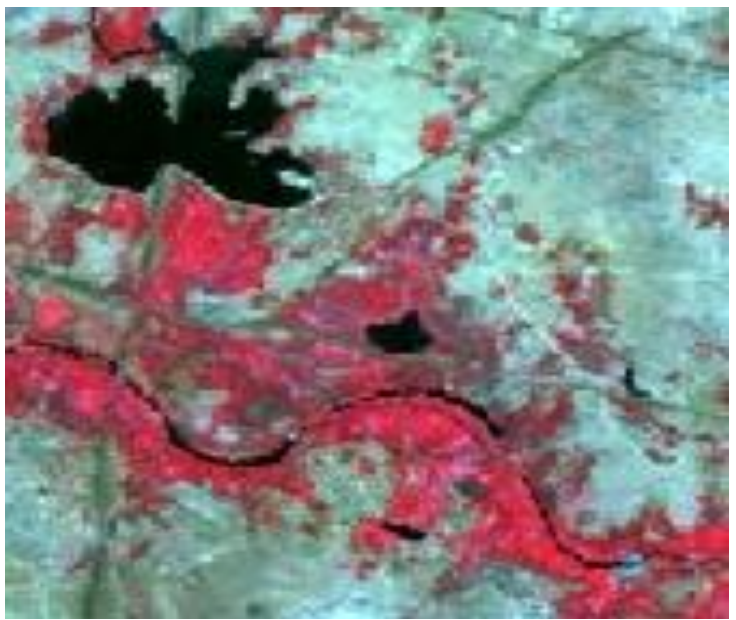

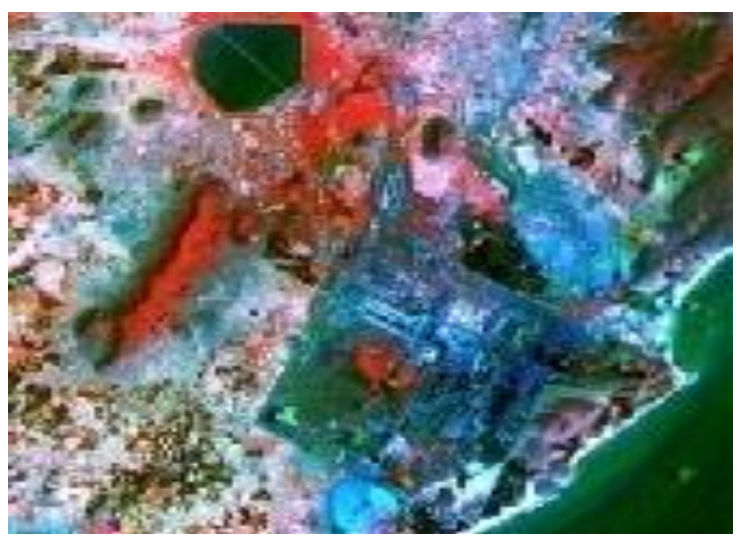
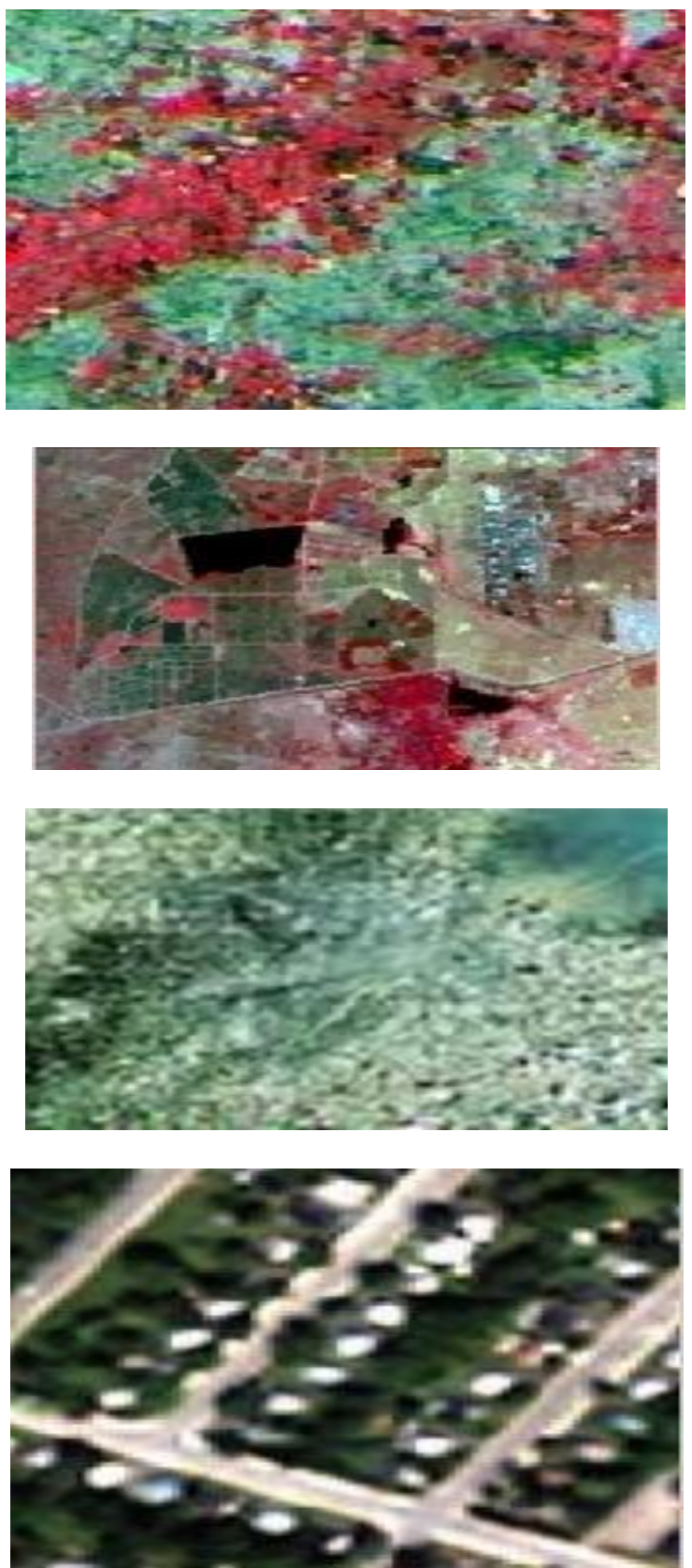

Figure 9: Proposed BFWN Fusion Technique at the Locations Hyderabad, Visakhapatnam,

Mahaboobnagar, Patancheru, Landsat-7 and QuickBird

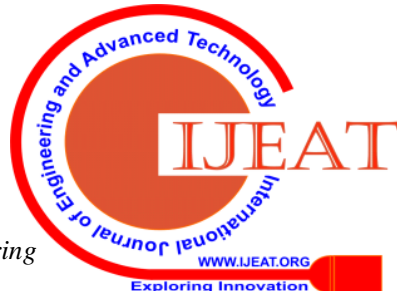




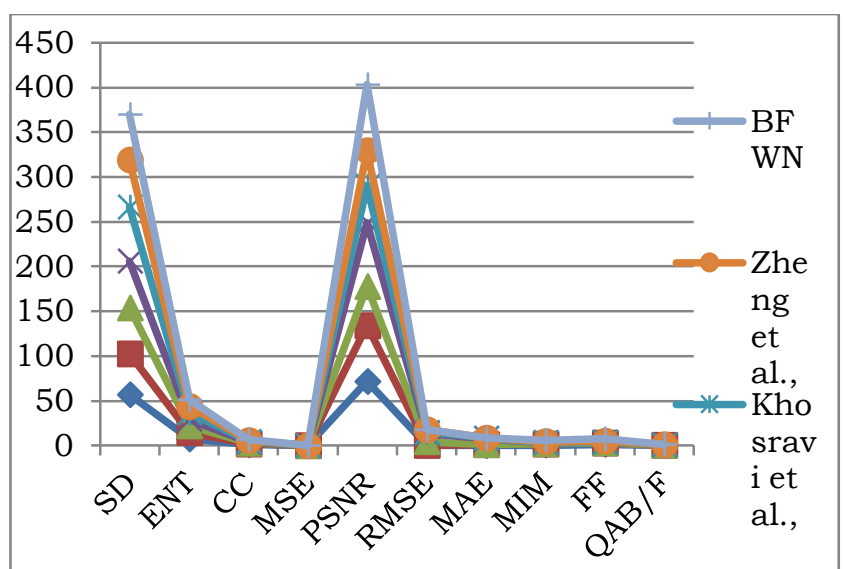

Figure 10: Comparative Analysis of the Proposed BFWN Method with the Other Existing Methods About the Quality Parameters

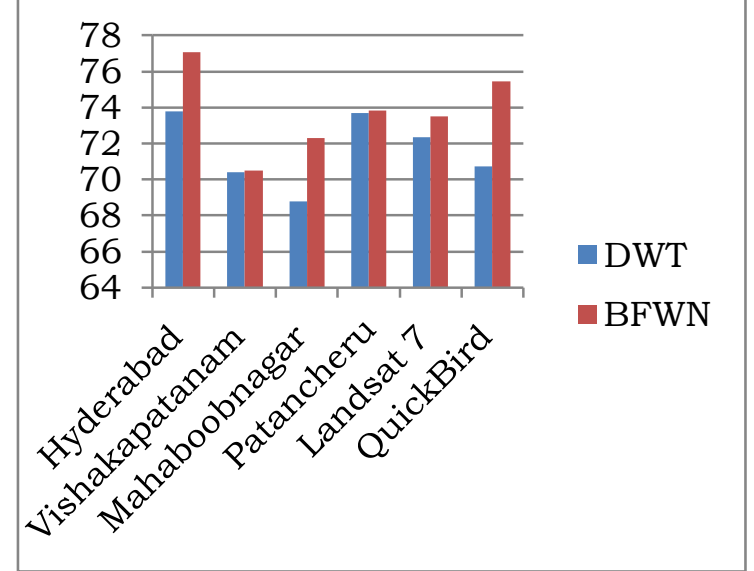

Figure 11: Comparing PSNR Values

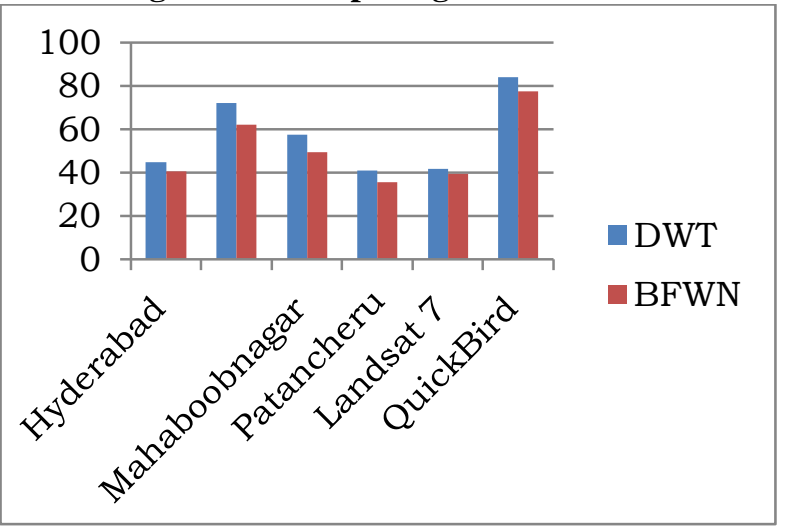

Figure 12: Comparing SD Values

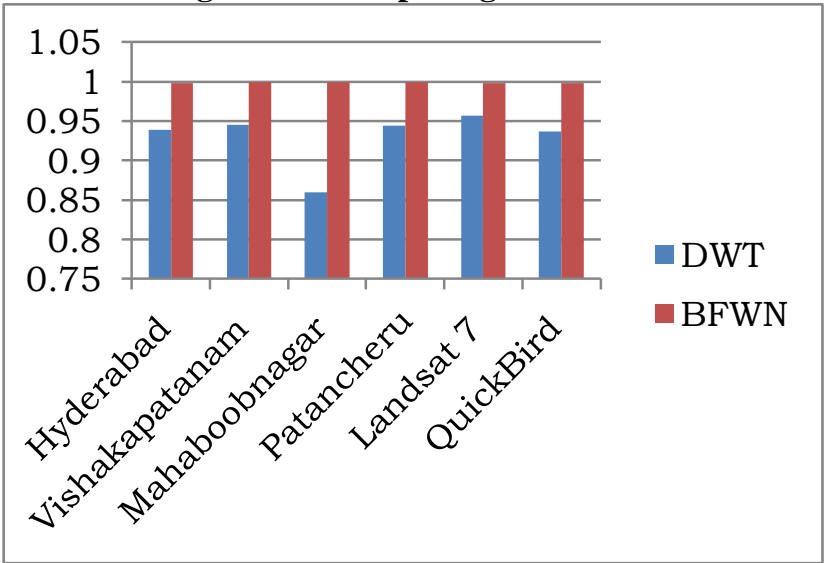

Figure 13: Comparing CC Values

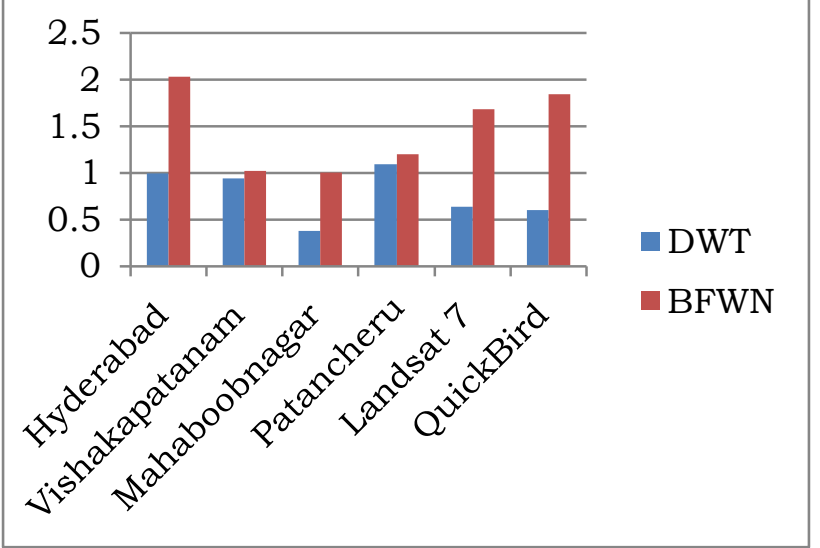

Figure 14: Comparing MIM Values

\section{CONCLUSION}

This paper describes the two fusion techniques i.e., DWT and the proposed BFWN are analysed by using the performance of the quality metrics. The experimental results illustrate that the proposed BFWN fusion algorithm gives encouraging results. The fused image looks more complete with minimum distortion i.e., most of the visual artefacts are eliminated. Thus, the fused image contains increased accuracy as well as reliability, which leads to better utility and robust performance and can be used for further interpretation. The proposed BFWN is used to make an intelligent decision and the adopted fusion rule selects fusion coefficients derived by using the discrete wavelet transform. A series of experimental results show that the BFWN algorithm is effective and provides good performance in fusing PAN and MS images.

Experimental results prove that the proposed method performs enhanced in preserving the image detail information than that of the other fusion methods. Hence, when discrete wavelet transform, which is a multi resolution analysis tool, integrated with the learning capabilities of neural network proved that it is ideally suited for image fusion purpose. The proposed method was tested with limited data set due to the unavailability of more satellite images. The BFWN method has numerous applications in the fields such as medical diagnosis, remote sensing, and machine vision and so on. This tool has the ability to assess the quality of the fusion result. This approach is more economic than designing a new sensor with both resolution characteristics, which is a sensor with high spatial and spectral resolution.

\section{REFERENCES}

1. Muhammad Fahim Uddin, Navarun Gupta, et al. Seven v's of big data understanding big data to extract value. In American Society for Engineering Education (ASEE Zone 1), 2014 Zone 1 Conference of the, pages 1-5. IEEE, 2014.

2. Fritz Venter and Andrew Stein. Images \& videos: really big data. Analytics Magazine, pages 14-47, 2012. 


\section{A METHODICAL BLOCK BASED FEATURE LEVEL IMAGE FUSION TECHNIQUE WITH WAVELET TRANSFORM USING NEURAL NETWORK FOR SATELLITE IMAGES}

3. U.S.N. Raju, Shibin George, V. Sairam Praneeth, Ranjeet Deo, Priyanka Jain, "Content Based Image Retrieval on Hadoop Framework", IEEE International Congress on Big Data, pp. 661 664, 2015.

4. Mu, Nan, Hongzhao Chen, Guijin Wang, Houyong Feng, and Feng Chen. "The online viewing system of the distributed gigapixel image." In Information Science and Technology (ICIST), 2016 Sixth International Conference on, pp. 132-136. IEEE, 2016.

5. Dong, Le, Zhiyu Lin, Yan Liang, Ling He, Ning Zhang, Qi Chen, Xiaochun Cao, and Ebroul Izquierdo. "A Hierarchical Distributed Processing Framework for Big Image Data." IEEE Transactions on Big Data 2, no. 4 (2016)

6. S.Mallat, A Wavelet Tour of Signal Processing, Academic Press, London, 1998.

7. Z. Li, Z. Jing, X. Yang, S. Sun, "Color transfer based Remote Sensing Image Fusion using nonseparable Wavelet Frame Transform, Pattern Recognition Lett”. 26(13), 2006-2014, 2005.

8. G.Hong and Y.Zhang, "Comparison and Improvement of wavelet-based Image fusion", Int. J. Remote Sens., 29:3, 673-69, Feb.2008.

9. Pohl C., Van Genderen J.L., "Multisensory image fusion in remote sensing: concepts, methods and applications". Int J. Remote Sens., vol.19, pp:823854, 1998.

10. Krista A., Yun Z., Peter D., "Wavelet based image fusion techniques - An introduction, review and comparison", ISPRS J. Photogram, Remote Sens., vol.62, pp: 249-263, 2007.

11. Yun Z., "Understanding image fusion", Photogram Eng. Remote Sens., vol.6, pp: 657661, 2004.

12. Shannon C.E., "A Mathematical Theory of Communication", Bell System Technical Journal, vol.27, pp: 379-423, October 1948.

13. George Casella, Lehmann E.L., Theory of Point Estimation. Springer, 1996

14. Abdul Basit Siddiqui, Arfan Jaffar M., Ayyaz Hussain, Anwar Mirza M., "Block-based Featurelevel Multi-focus Image Fusion", 5th International Conference on Future Information Technology (Future Tech), IEEE, 978-1-42446949-9/10, 2010.

15. Yan Luo, Rong Liu, Yu Feng Zhu - "FUSION OF REMOTE SENSING IMAGE BASE ON THE PCA+ATROUS WAVELET TRANSFORM", The International Archives of the Photogrammetric, Remote Sensing and Spatial Information Sciences, vol. XXXVII, Part B7, Beijing, 2008.

16. Yuhendra, "Wavelet PCA Based Images Fusion Techniques And Quality Assessment"

17. Maziyar Khosravi, Mazaheri Amin, "BLOCK FEATURE BASED IMAGE FUSION USING MULTI WAVELET TRANSFORMS", International Journal of Engineering Science and Technology, vol.3, no.8, August 2011

18. Hong Zheng, Dequan Zheng, Yanxiang HU., Sheng LI., "Study on the Optimal Parameters of Image Fusion Based on Wavelet Transform", Journal of Computational Information Systems, vol.6, no.1, pp:131-137, 2010 